\title{
Therapeutic and prophylactic vaccines to counteract fentanyl use disorders and toxicity
}

Christine Robinson"1, Valeria Gradinati', Fatima Hamid'1, Carly Baehr ${ }^{1,2}$, Bethany Crouse ${ }^{1}$, Saadyah Averick ${ }^{3}$, Marina Kovaliov $^{3}$, Danni Harris ${ }^{4}$, Scott Runyon ${ }^{4}$, Federico Baruffaldi5,6, Mark LeSage ${ }^{6}$, Sandra Comer ${ }^{7}$ and Marco Pravetoni ${ }^{1}, 8^{*}$.

${ }^{1}$ Department of Pharmacology, University of Minnesota Medical School, Minneapolis, Minnesota, 55455, USA. ${ }^{2}$ Department of Veterinary Population Medicine, University of Minnesota Veterinary School, Minneapolis, Minnesota, 55455, USA. ${ }^{3}$ Allegheny Health Network, Neuroscience Research Institute, Pittsburgh, Pennsylvania, 15212, USA. ${ }^{4}$ RTI International, Raleigh, North Carolina, 27616, USA. ${ }^{5}$ Coherus Biosciences, Redwood City, California, 94065 (current address). ${ }^{6}$ Department of Medicine, Hennepin Healthcare Research Institute, Minneapolis, Minnesota 55415, USA. ${ }^{7}$ Department of Psychiatry, Columbia University Irving Medical Center, and the New York State Psychiatric Institute, New York, New York 10027, USA. ${ }^{8}$ Center for Immunology, University of Minnesota Medical School, Minneapolis, Minnesota, 55455, USA.

\section{Corresponding author.}

*Marco Pravetoni, PhD. E-mail: prave001@umn.edu.

\section{Content}

Supplemental Methods: analytical chemistry

Supplemental Table 1

Supplemental Table 2

Supplemental Table 3

Supplemental Table 4

Supplemental Figure 1

Supplemental Figure 2

Supplemental Figure 3

Supplemental Figure 4

Supplemental Figure 5

Supplemental Figure 6 


\section{Analysis of fentanyl concentrations in tissue: extraction and LC-MS conditions.}

Preparation of brain samples. Brain tissue was homogenized using Agilent ceramic beads for 4 min with a Beadblaster 24 homogenizer (Benchmark Scientific, Sayreville, NJ) at $7 \mathrm{~m} / \mathrm{s}$, then centrifuged briefly to reduce bubbles. The homogenate was transferred to a cryogenic tube and placed at $-20^{\circ} \mathrm{C}$ until time of extraction.

Sample extraction. Extraction of serum, brain homogenate, and standards was performed at $4^{\circ} \mathrm{C}$. For standards, $20 \mu \mathrm{L}$ of stock calibrator solution was added to $180 \mu \mathrm{L}$ of fetal bovine serum. $200 \mu \mathrm{L}$ of sample was used for extraction with $20 \mu \mathrm{L}$ of internal standard solution added to all samples. $600 \mu \mathrm{L}$ of cold LCMS grade Acetonitrile was added to all tubes to precipitate proteins and then centrifuged at 8,609 $\mathrm{xg}$ for $10 \mathrm{~min}$. Supernatant was transferred to glass tubes, evaporated to $200 \mu \mathrm{L}$ on a Cerex 48 Sample Concentrator (Tecan, Männedorf, Switzerland), and then diluted 1:1 with $0.1 \mathrm{M}$ phosphate buffer. Extraction was performed using Bond Elut Plexa PCX, 3mL extraction cartridges (Agilent, Santa Clara, CA). Cartridges were first washed with $500 \mu \mathrm{L}$ methanol and then samples were loaded onto cartridges. Cartridges were washed in series, first using $2 \mathrm{~mL}$ of $2 \%$ formic acid followed by $3 \mathrm{~mL} 70 \% / 30 \%$ methanol: $2 \%$ formic acid. Cartridges were dried on a Cerex 48 Positive Pressure Manifold (Tecan, Männedorf, Switzerland), placed above fresh tubes to elute samples using $3 \mathrm{~mL}$ of $5 \%$ ammonium hydroxide in acetonitrile, and dried on the sample concentrator. Samples were reconstituted in $50 \mu \mathrm{L}$ LCMS grade water, $0.1 \%$ ammonium formate, $0.01 \%$ LCMS grade formic acid (mobile phase A).

LCMS/MS conditions. Two microliters of sample were injected onto a reversed phase Agilent (Santa Clara, CA) Zorbax Eclipse plus C18 $(2.1 \mathrm{~mm} \times 50 \mathrm{~mm}$ i.d., $1.8 \mu \mathrm{m})$ column. The LCMS/MS system consisted of an Agilent G6470A triple quadrupole with an Infinity II 1290 G7116B Multicolumn Thermostat, G7120A High Speed Quad Pumps, G7267B Multisampler. The samples were kept at $4^{\circ} \mathrm{C}$ during injection. Gradient elution was performed with a mixture of mobile phase $A$ and methanol, $0.01 \%$ formic acid (mobile phase $B$ ) as follows: $0-0.5$ min $5 \%$ mobile phase $B, 0.5-2.25 \min 15 \rightarrow 50 \%$ mobile phase $B, 2.25-4.0$ min $50 \rightarrow 95 \%$ mobile phase $B, 4.0-6.095 \%$ mobile phase $B$. The flow rate was kept at a constant $0.40 \mathrm{~mL} / \mathrm{min}$ and the total run time was 6 min. The autosampler needle was washed with a solution of $50 \%$ LCMS-grade-water, $25 \%$ acetonitrile, $25 \%$ methanol following each sample injection. 
Electrospray ionization was achieved by Agilent Jet Stream high sensitivity ion source in the positive ion mode. Instrument settings were: gas temperature $300^{\circ} \mathrm{C}$, gas flow $5 \mathrm{~L} / \mathrm{min}$, nebulizer pressure 45 psi, sheath gas temperature $250^{\circ} \mathrm{C}$, sheath gas flow $11 \mathrm{~L} / \mathrm{min}$, capillary $3500 \mathrm{~V}$, and nozzle $900 \mathrm{~V}$. Data acquisition and peak integration were interfaced to a computer workstation using Mass Hunter (Tokyo, Japan). LCMS/MS in the SIM mode was used to identify the appropriate ions to monitor: fentanyl $337.2-188.1$, secondary $337.2-105.1$, Fentanyl-d5 342.3 - 188.1, secondary 342.3 - 105.1 . 
Supplemental Table 1. Characterization of conjugates containing the $F_{1-6}$ hapten series.

\begin{tabular}{|c|c|c|c|}
\hline Conjugate & $\begin{array}{c}\text { MW (MALDI- } \\
\text { TOF) }\end{array}$ & $\begin{array}{c}\text { Calculated } \\
\text { Haptenization } \\
\text { Ratio }\end{array}$ & Precipitate/Aggregate \\
\hline $\mathrm{F}_{1-\mathrm{BSA}}$ & 81297.47 & 23 & No \\
\hline $\mathrm{F}_{1-\mathrm{SKLH}}$ & $\mathrm{N} / \mathrm{A}$ & $\mathrm{N} / \mathrm{A}$ & Yes \\
\hline $\mathrm{F}_{1}-\mathrm{CRM}_{1}$ & 70049.77 & 18,17 & Slightly \\
\hline $\mathrm{F}_{1}-\mathrm{CRM}_{2}$ & 66578.75 & 12,17 & Slightly \\
\hline $\mathrm{F}_{2}$-BSA & 78185.07 & 23 & No \\
\hline $\mathrm{F}_{2}$-sKLH & $\mathrm{N} / \mathrm{A}$ & $\mathrm{N} / \mathrm{A}$ & No \\
\hline $\mathrm{F}_{3}-\mathrm{BSA}$ & 70713.41 & 9 & No \\
\hline $\mathrm{F}_{3}$-sKLH & $\mathrm{N} / \mathrm{A}$ & $\mathrm{N} / \mathrm{A}$ & No \\
\hline $\mathrm{F}_{3}-\mathrm{CRM}_{2}$ & 61786.37 & 6 & Yes \\
\hline $\mathrm{F}_{4}-\mathrm{BSA}$ & 69473.86 & 7 & No \\
\hline $\mathrm{F}_{4-\mathrm{SKLH}}$ & $\mathrm{N} / \mathrm{A}$ & $\mathrm{N} / \mathrm{A}$ & No \\
\hline $\mathrm{F}_{5}-\mathrm{BSA}$ & 74246.50 & 17 & No \\
\hline $\mathrm{F}_{5}$-sKLH & $\mathrm{N} / \mathrm{A}$ & $\mathrm{N} / \mathrm{A}$ & Yes \\
\hline $\mathrm{F}_{5}-\mathrm{CRM}_{2}$ & 63243.98 & 10 & Yes \\
\hline $\mathrm{F}_{6}-\mathrm{BSA}$ & 74060.00 & 17 & No \\
\hline $\mathrm{F}_{6}$-sKLH & $\mathrm{N} / \mathrm{A}$ & $\mathrm{N} / \mathrm{A}$ & Yes \\
\hline $\mathrm{F}_{6}-\mathrm{CRM}_{2}$ & 62145.96 & 7 & Slightly \\
\hline
\end{tabular}

$\mathrm{F}_{1-6}$ haptens were conjugated to subunit $\mathrm{KLH}(\mathrm{SKLH})$, and either $\mathrm{CRM}_{1}$ (E. coli-expressed CRM from FinaBioSolutions) or $\mathrm{CRM}_{2}$ (Pfenex) depending on product availability. 
Supplemental Table 2. Characterization of antibodies' affinity for fentanyl, its analogs, and selected offtarget opioids. Sera from A) mice, and B-C) rats immunized with conjugates containing fentanyl-based haptens conjugated to either sKLH, $\mathrm{CRM}_{1}$, or $\mathrm{CRM}_{2}$. Conjugates were adsorbed on aluminum adjuvant and injected i.m..

A. Mouse

\begin{tabular}{|c|c|c|c|}
\hline $\begin{array}{c}\text { Vaccine } \\
\text { Formulation }\end{array}$ & Coating & $\begin{array}{l}\text { Fentanyl IC } C_{50}(\mathrm{nM}) \\
\text { Individual value }\end{array}$ & $\begin{array}{c}\text { Fentanyl IC } \\
\text { Mean } \pm \text { SEM }\end{array}$ \\
\hline $\mathrm{F}_{1-\mathrm{SKLH}}$ & $F_{1-B S A}$ & $204.4,61.8$ & $133.1 \pm 71.3$ \\
\hline $\mathrm{F}_{1-\mathrm{SKLH}}$ & $F_{2}-B S A$ & $155.3,598.6$ & $377.0 \pm 221.7$ \\
\hline $\mathrm{F}_{1-\mathrm{SKLH}}$ & $F_{3}-B S A$ & $11.8,5.3$ & $8.55 \pm 3.25$ \\
\hline $\mathrm{F}_{1-\mathrm{SKLH}}$ & $\mathrm{F}_{1}-\mathrm{BSA}$ & $45.8,3.30,22.7,15.0,80.6,68.5$ & $39.3 \pm 12.6$ \\
\hline$F_{2-S K L H}$ & $F_{2}-B S A$ & 282.9 & 282.9 \\
\hline $\mathrm{F}_{2}-\mathrm{CRM}_{1}$ & $\mathrm{~F}_{2}-\mathrm{BSA}$ & 891.2 & 891.2 \\
\hline $\mathrm{F}_{3}$-sKLH & $F_{3}-B S A$ & 140.3 & 140.3 \\
\hline $\mathrm{F}_{3}-\mathrm{CRM}_{1}$ & $\mathrm{~F}_{3}-\mathrm{BSA}$ & 67.9 & 67.9 \\
\hline $\mathrm{F}_{4}$-sKLH & $\mathrm{F}_{1}-\mathrm{BSA}$ & $>100 \mu \mathrm{M}$ (undetectable) & $>100 \mu \mathrm{M}$ (undetectable) \\
\hline $\mathrm{F}_{5}$-sKLH & $F_{1}-B S A$ & $14.1,57.5$ & $35.7 \pm 21.6$ \\
\hline $\mathrm{F}_{6}-\mathrm{CRM}_{2}$ & $F_{1}-B S A$ & 5.8468 .1 & $36.97 \pm 31.13$ \\
\hline
\end{tabular}

B. Rat

\begin{tabular}{|c|c|c|c|c|c|c|}
\hline $\begin{array}{c}\text { Vaccine } \\
\text { Formulation }\end{array}$ & Coating & $\begin{array}{l}\text { Fentanyl } \\
I^{\prime} C_{50}(\mu \mathrm{M})\end{array}$ & $\begin{array}{l}\text { Fentanyl } \\
I^{\prime} C_{50}(\mathrm{nM})\end{array}$ & $\begin{array}{l}\text { Sufentanil } \\
I C_{50}(\mu M)\end{array}$ & $\begin{array}{l}\text { Alfentanil } \\
\mathrm{IC}_{50}(\mu \mathrm{M})\end{array}$ & $\begin{array}{l}\text { Remifentanil } \\
\text { IC }_{50}(\mu \mathrm{M})\end{array}$ \\
\hline $\begin{array}{c}\mathrm{F}_{1}-\mathrm{SKLH} \\
\left(1^{\mathrm{st}} \text { cohort }\right)\end{array}$ & $\mathrm{F}_{1-\mathrm{BSA}}$ & $\begin{array}{l}0.0142 \pm \\
0.00414\end{array}$ & $14.2 \pm 4.14$ & $17.78 \pm 0.59$ & $60.23 \pm 39.77$ & $64.72 \pm 35.29$ \\
\hline $\mathrm{F}_{1}-\mathrm{CRM}_{1}$ & $\mathrm{~F}_{1-\mathrm{BSA}}$ & $\begin{array}{c}0.5303 \\
0.530 \pm 1.67\end{array}$ & $530 \pm 167$ & $14.60 \pm 6.86$ & $>100$ & $>100$ \\
\hline $\mathrm{F}_{1}-\mathrm{CRM}_{2}$ & $\mathrm{~F}_{1}-\mathrm{BSA}$ & $\begin{array}{c}0.1132 \\
0.113 \pm 0.0634\end{array}$ & $113.2 \pm 63.4$ & $65.13 \pm 34.88$ & $66.55 \pm 33.46$ & $>100$ \\
\hline $\mathrm{F}_{2}$-sKLH & $\mathrm{F}_{2}-\mathrm{BSA}$ & $\begin{array}{c}0.0248 \\
0.0248 \pm \\
0.0091\end{array}$ & $24.8 \pm 9.1$ & $14.68 \pm 6.84$ & 100 & $>100$ \\
\hline $\mathrm{F}_{3-\mathrm{SKLH}}$ & $\mathrm{F}_{3}$-BSA & $\begin{array}{c}0.0696 \\
0.0696 \pm \\
0.0089\end{array}$ & $69.6 \pm 8.9$ & $10.55 \pm 0.26$ & 100 & $>100$ \\
\hline $\begin{array}{c}\mathrm{F}_{1-S K L H} \\
\text { (2nd cohort) }\end{array}$ & $\mathrm{F}_{1-\mathrm{BSA}}$ & $\begin{array}{c}0.0091,0.0915 \\
0.0503 \pm \\
0.0412\end{array}$ & $50.3 \pm 41.2$ & $>100 \mu M$ & $>100 \mu M$ & $>100 \mu M$ \\
\hline $\mathrm{F}_{4-\mathrm{SKLH}}$ & $\mathrm{F}_{1}-\mathrm{BSA}$ & $>100 \mu M$ & $>100 \mu \mathrm{M}$ & $>100 \mu \mathrm{M}$ & $>100 \mu \mathrm{M}$ & $>100 \mu \mathrm{M}$ \\
\hline $\mathrm{F}_{5}$-sKLH & $F_{1}-B S A$ & $>100 \mu \mathrm{M}$ & $>100 \mu \mathrm{M}$ & $>100 \mu \mathrm{M}$ & $>100 \mu \mathrm{M}$ & $>100 \mu \mathrm{M}$ \\
\hline $\mathrm{F}_{6}-\mathrm{CRM}_{2}$ & $F_{1}-B S A$ & $>100 \mu M$ & $>100 \mu \mathrm{M}$ & $>100 \mu \mathrm{M}$ & $>100 \mu \mathrm{M}$ & $>100 \mu M$ \\
\hline
\end{tabular}

Polyclonal sera from rats immunized with the series of conjugates containing $\mathrm{F}_{1-6}$ haptens were analyzed by competitive binding ELISA, and when possible compared to a monoclonal antibody (mAb) isolated from mice immunized with $\mathrm{F}_{1}$-sKLH. The anti- $\mathrm{F}_{1} \mathrm{mAb}$ shows $\mathrm{K}_{d}$ of $1.59-0.53 \mathrm{nM}$ for fentanyl as measured by biolayer interferometry (BLI). The anti- $\mathrm{F}_{1}$ mAb has been previously described [1].

C. Off-target opioids

\begin{tabular}{|c|c|c|c|c|c|}
\hline $\begin{array}{c}\text { Vaccine } \\
\text { Formulation }\end{array}$ & Coating & $\begin{array}{c}\text { Buprenorphine } \\
\text { IC }_{50}(\mathrm{mM})^{\star}\end{array}$ & $\begin{array}{l}\text { Naloxone } \\
I_{50}(\mathrm{mM})\end{array}$ & $\begin{array}{l}\text { Naltrexone } \\
I_{50}(\mathrm{mM})\end{array}$ & $\begin{array}{c}\text { Methadone } \\
I_{50}(\mathrm{mM})\end{array}$ \\
\hline $\mathrm{F}_{1-\mathrm{sKLH}}$ & $F_{1}-B S A$ & $>0.3$ & $>10$ & $>10$ & $>10$ \\
\hline$F_{2}$-sKLH & $F_{1}-B S A$ & $>0.3$ & $0.1,1$ & $1,>10$ & $>10,1.1$ \\
\hline $\mathrm{F}_{3}$-sKLH & $\mathrm{F}_{1}-\mathrm{BSA}$ & $>0.3$ & $9.2,>10$ & $>10$ & $>10$ \\
\hline $\mathrm{F}_{4-\mathrm{SKLH}}$ & $F_{1}-B S A$ & $>0.3$ & $>10$ & $>10$ & $>10$ \\
\hline $\mathrm{F}_{5}$-sKLH & $F_{1}-B S A$ & $>0.3$ & $>10$ & $>10$ & $>16$ \\
\hline $\mathrm{F}_{6}-\mathrm{CRM}_{2}$ & $F_{1-B S A}$ & $>0.3$ & $>10$ & $1.9,2$ & $>10$ \\
\hline
\end{tabular}

${ }^{*}$ The maximal concentration of buprenorphine tested was $0.3 \mathrm{mM}$ because of the commercially available drug stock concentration. The maximal concentrations of naloxone, naltrexone, and methadone were $10 \mathrm{mM}$. 
Supplemental Table 3. Absolute values for number of infusions (mean \pm SEM) at each fentanyl unit dose in $\mathrm{CRM}_{1}$ and $\mathrm{F}_{1}-\mathrm{CRM}_{1}$ immunized rats during unit dose reduction.

\begin{tabular}{|c|c|c|c|c|c|c|c|}
\hline & \multicolumn{3}{|c|}{ CRM $_{\mathbf{1}}$} & \multicolumn{5}{c|}{ F $_{\mathbf{1}}-\mathbf{C R M}_{\mathbf{1}}$} \\
\hline Fentanyl Dose $(\boldsymbol{\mu g} / \mathbf{k g})$ & Mean & $\mathbf{S E M}$ & ${ }^{*} \boldsymbol{p}$ & Mean & SEM & ${ }^{*} \boldsymbol{p}$ & ${ }^{\#} \boldsymbol{p}$ \\
\hline 0.00 & 16.3 & 3.1 & & 9.5 & 0.7 & & $\mathrm{~ns}$ \\
\hline 0.25 & 58.5 & 11.1 & $<0.05$ & 8.5 & 3.4 & $\mathrm{~ns}$ & $>0.01$ \\
\hline 0.50 & 55.5 & 7.3 & $<0.05$ & 34.5 & 22.8 & $\mathrm{~ns}$ & $\mathrm{~ns}$ \\
\hline 0.75 & 48.7 & 6.1 & $<0.05$ & 27.6 & 13.9 & $\mathrm{~ns}$ & $\mathrm{~ns}$ \\
\hline 1.00 & 45.2 & 5.2 & $<0.05$ & 47.7 & 11.7 & $<0.01$ & $\mathrm{~ns}$ \\
\hline
\end{tabular}

Statistical $p$ values based on analysis of log transformed data. ${ }^{*}$ Compared to $0.00 \mu \mathrm{g} / \mathrm{kg}$. \#Compared to $\mathrm{CRM}_{1}$. Ns= Not significant. 
Supplemental Table 4. Qualitative characterization of in vivo efficacy of conjugates containing the $F_{1-6}$ hapten series. Scoring: -- = Not tested; $0=$ no effect; $1=$ low effect; $2=$ medium effect; $3=$ high effect.

\begin{tabular}{|c|c|c|c|c|c|c|c|c|c|c|}
\hline Vaccine & $\begin{array}{c}\text { Drug } \\
\text { Increase } \\
\text { [Serum] }\end{array}$ & $\begin{array}{c}\text { Drug } \\
\text { Decrease } \\
\text { [Brain] }\end{array}$ & $\begin{array}{l}\text { Fentanyl } \\
\text { MPE\% }\end{array}$ & $\begin{array}{c}\mathrm{SaO}_{2} \% \\
(0.075 \\
\mathrm{mg} / \mathrm{kg})\end{array}$ & $\begin{array}{c}\text { Heart } \\
\text { Rate } \\
(0.075 \\
\mathrm{mg} / \mathrm{kg})\end{array}$ & $\begin{array}{c}\mathrm{SaO}_{2} \% \\
(0.1 \\
\mathrm{mg} / \mathrm{kg})\end{array}$ & $\begin{array}{c}\text { Heart } \\
\text { Rate } \\
(0.1 \\
\mathrm{mg} / \mathrm{kg})\end{array}$ & $\begin{array}{l}\text { Sufentanil } \\
\text { MPE\% }\end{array}$ & $\begin{array}{l}\text { Total } \\
\text { Score }\end{array}$ & FSA \\
\hline$F_{1-s K L H}$ & 2 & 2 & 1 & 3 & 1 & 1 & 1 & 0 & 11 & -- \\
\hline $\mathrm{F}_{1}-\mathrm{CRM}_{1}$ & -- & 3 & 3 & 3 & 3 & 3 & 3 & 1 & 19 & 2 \\
\hline $\mathrm{F}_{1}-\mathrm{CRM}_{2}$ & -- & 3 & 3 & 3 & 3 & 3 & 3 & 3 & 21 & -- \\
\hline $\mathrm{F}_{2-\mathrm{SKLH}}$ & -- & 3 & 3 & 3 & 3 & 3 & 3 & 3 & 21 & -- \\
\hline $\mathrm{F}_{3-\mathrm{SKLH}}$ & -- & 3 & 3 & 3 & 3 & 3 & 3 & 1 & 19 & -- \\
\hline $\mathrm{F}_{3}-\mathrm{CRM}_{2}{ }^{*}$ & -- & 3 & 3 & -- & -- & -- & -- & -- & 6 & -- \\
\hline $\mathrm{F}_{4-\mathrm{SKLH}}$ & 0 & 1 & 3 & -- & -- & 3 & 3 & 2 & 12 & -- \\
\hline $\mathrm{F}_{5-\mathrm{SKLH}}$ & 2 & 2 & 3 & -- & -- & 3 & 3 & 2 & 15 & -- \\
\hline $\mathrm{F}_{6}-\mathrm{CRM}_{2}{ }^{* *}$ & 3 & 3 & 3 & -- & -- & 3 & 3 & 3 & 18 & -- \\
\hline
\end{tabular}

$\mathrm{F}_{1-6}$ haptens were conjugated to subunit $\mathrm{KLH}(\mathrm{sKLH})$, and either $\mathrm{CRM}_{1}$ (E. coli-expressed CRM from FinaBio) or $\mathrm{CRM}_{2}$ (Pfenex) depending on product availability. Scoring excludes FSA, as only one conjugate was used for those experiments. Symbol: ${ }^{*}$ indicates conjugates tested only in mice. Note: sufentanil oximetry scores for $\mathrm{SaO}_{2} \%$ and heart rate were 0 and therefore not included in this table. **effective against fentanyl-induced breath rate reduction after $1 \mathrm{mg} / \mathrm{kg}$ fentanyl challenge (Supplemental Figure 6). 
Supplemental Figure 1. Analysis and purification of the $F_{2}, F_{3}, F_{4}, F_{5}$, and $F_{6}$ haptens by HPLC.

Panel A ( $\mathbf{F}_{2}$ and $\left.\mathbf{F}_{3}\right)$. Spectra were collected using a 1260 Infinity Isocratic Pump with a ZirChrom®-PBD column (50 $\mathrm{mm} \times 2.1 \mathrm{~mm}$ i.d., 3 micron). The mobile phase was acetonitrile/10 mM ammonium acetate $(\mathrm{v} / \mathrm{v}=45 / 55)$ with $0.1 \mathrm{mM}$ citrate $\left(\mathrm{pH} \mathrm{4.4)}\right.$ and the temperature was $25^{\circ} \mathrm{C}$. The flow rate was set at $0.3 \mathrm{ml} / \mathrm{min}$. Purity: $\mathrm{F}_{2}=$ $97.0 \%$, and $F_{3}=97.7 \%$.
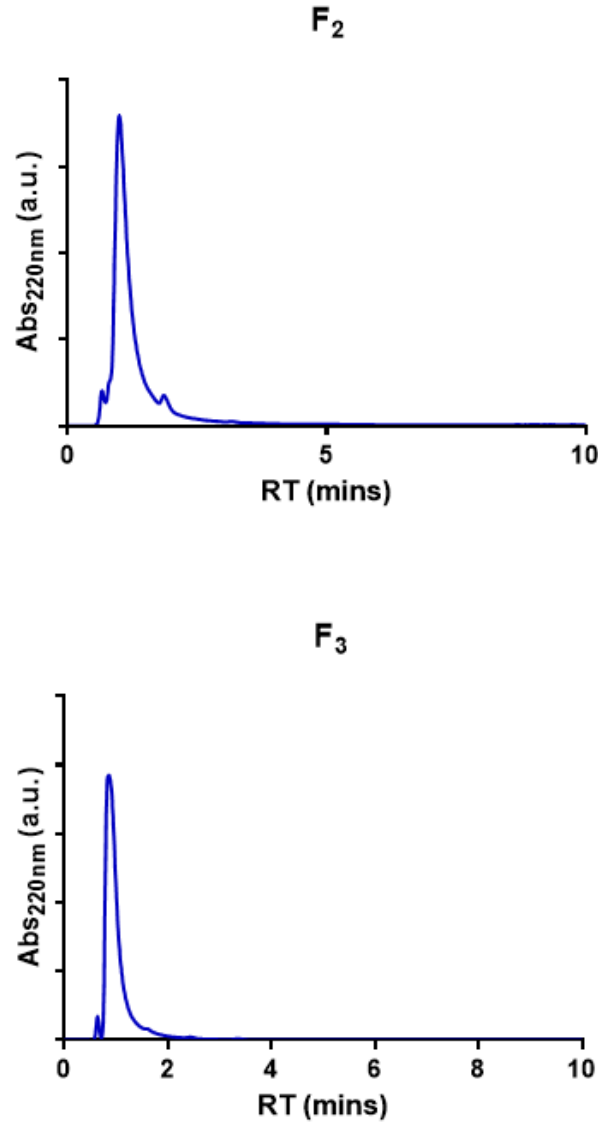
Panel B $\left(F_{4}\right)$. Purity $=95 \%$.

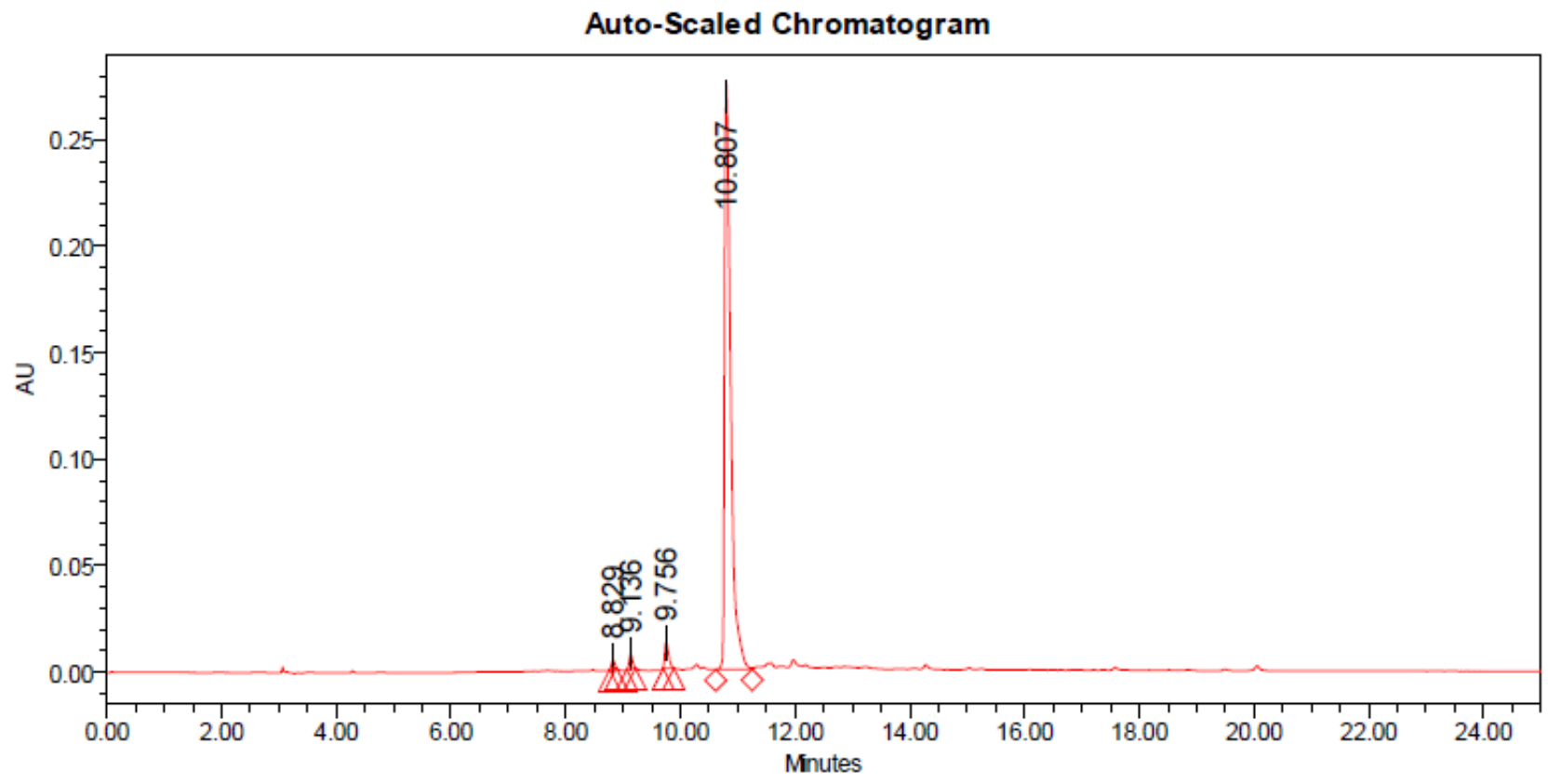

Peak Results
\begin{tabular}{|r|r|r|r|r|}
\hline & RT & Area & $\%$ Area & Height \\
\hline 1 & 8.829 & 14291 & 0.66 & 3702 \\
\hline 2 & 9.136 & 27917 & 1.29 & 6745 \\
\hline 3 & 9.756 & 57694 & 2.66 & 12231 \\
\hline 4 & 10.807 & 2065695 & 95.39 & 281511 \\
\hline
\end{tabular}

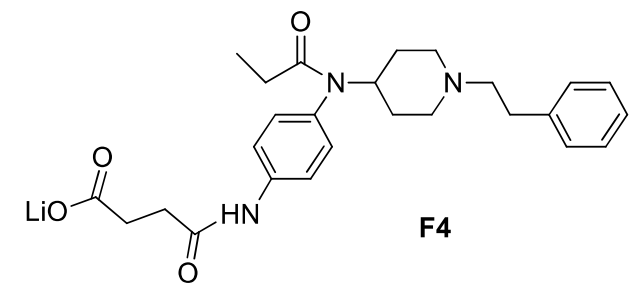


Panel C $\left(F_{5}\right)$. Purity $=96 \%$.

Auto-Scaled Chromatogram

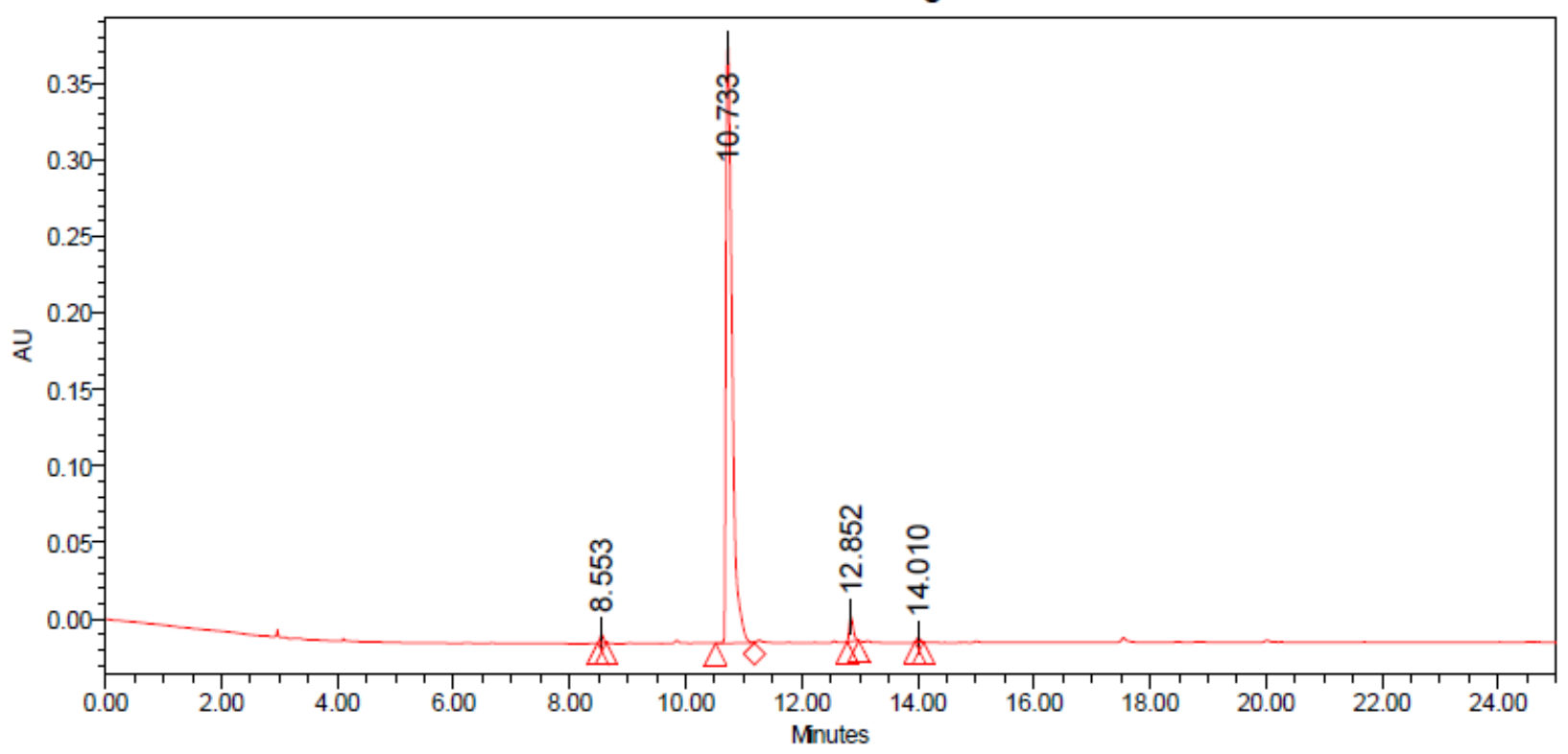

Peak Results

\begin{tabular}{|r|c|r|r|r|}
\hline & RT & Area & \% Area & Height \\
\hline 1 & 8.553 & 22245 & 0.75 & 5407 \\
\hline 2 & 10.733 & 2861527 & 96.36 & 391999 \\
\hline 3 & 12.852 & 73592 & 2.48 & 15839 \\
\hline 4 & 14.010 & 12268 & 0.41 & 2758 \\
\hline
\end{tabular}

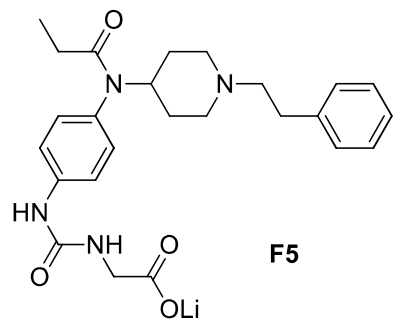


Panel D $\left(\mathbf{F}_{6}\right)$. Purity $=100 \%$.

\section{Auto-Scaled Chromatogram}

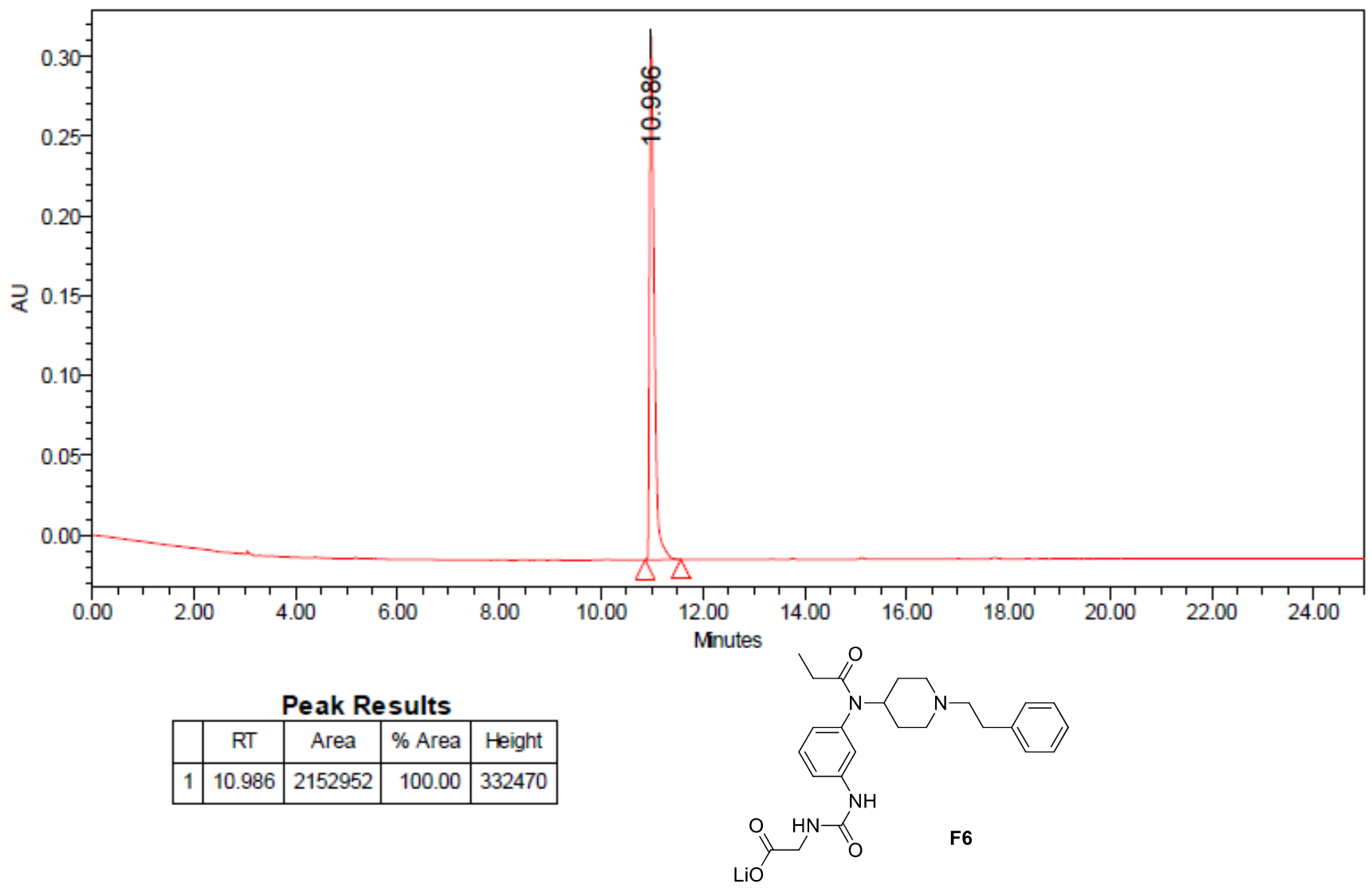


Supplemental Figure 2. Core structure homology between fentanyl and fentanyl-based haptens.

\section{FENTANYL}<smiles>CCC(=O)N(c1ccccc1)C1CCN(CCc2ccccc2)CC1</smiles>

$\mathbf{F}_{1}$

$\mathbf{F}_{2}$

$\mathbf{F}_{3}$

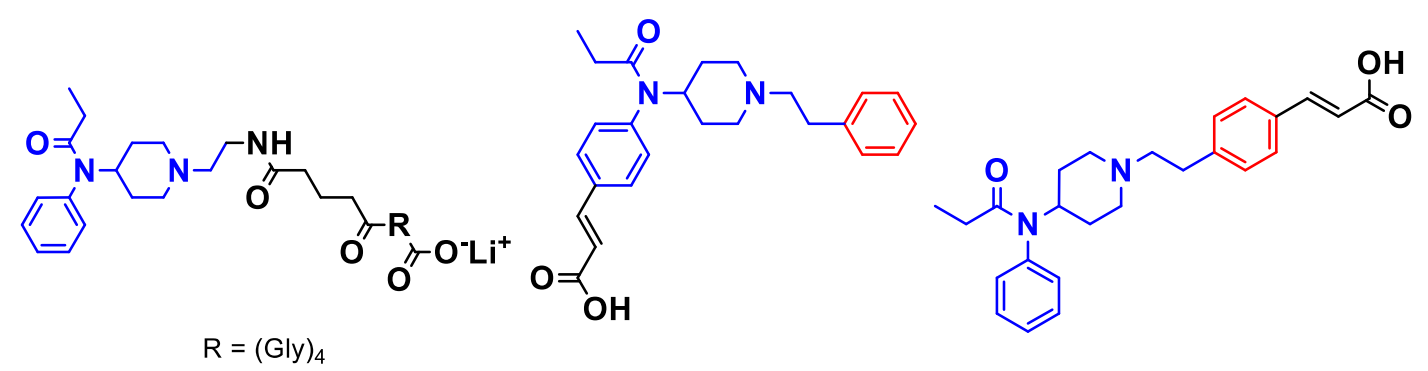

$\mathbf{F}_{4}$

$\mathbf{F}_{5}$

$F_{6}$
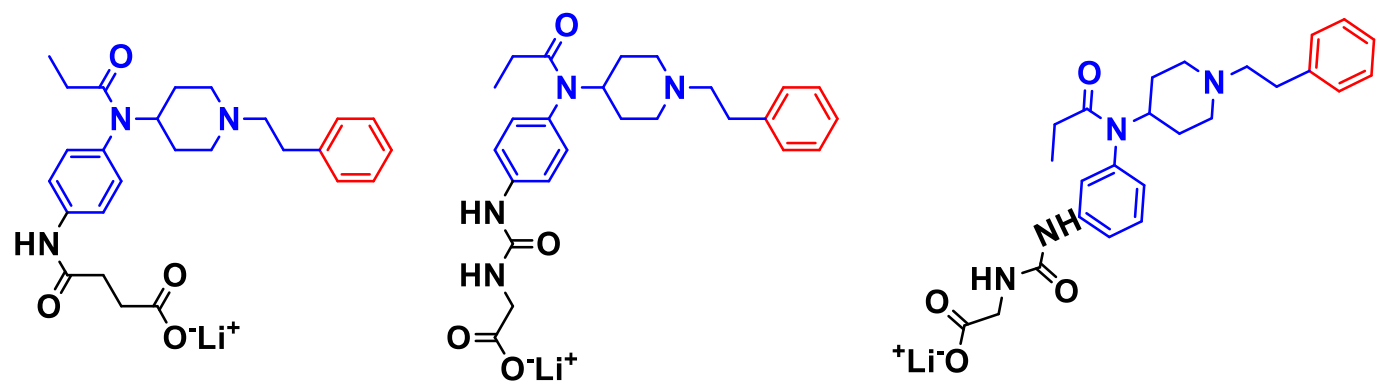
Supplemental Figure 3. Representative MALDI-TOF traces of conjugates and unconjugated carrier proteins. A) BSA and $F_{1}$-BSA with an haptenization ration (HR) of 23. Representative Dynamic Light Scattering (DLS) traces of B) $\left.F_{1}-s K L H, C\right) F_{1}-C_{R M}$, and D) $F_{1}-C_{2} M_{2}$. Panels $B-D$ ) show conjugates at $T=0$ and 1 month after storage at $+4^{\circ} \mathrm{C}$. $\mathrm{Ml}$ is mean diameter of the intensity distribution, $\mathrm{PDI}$ is the polydispersity index.
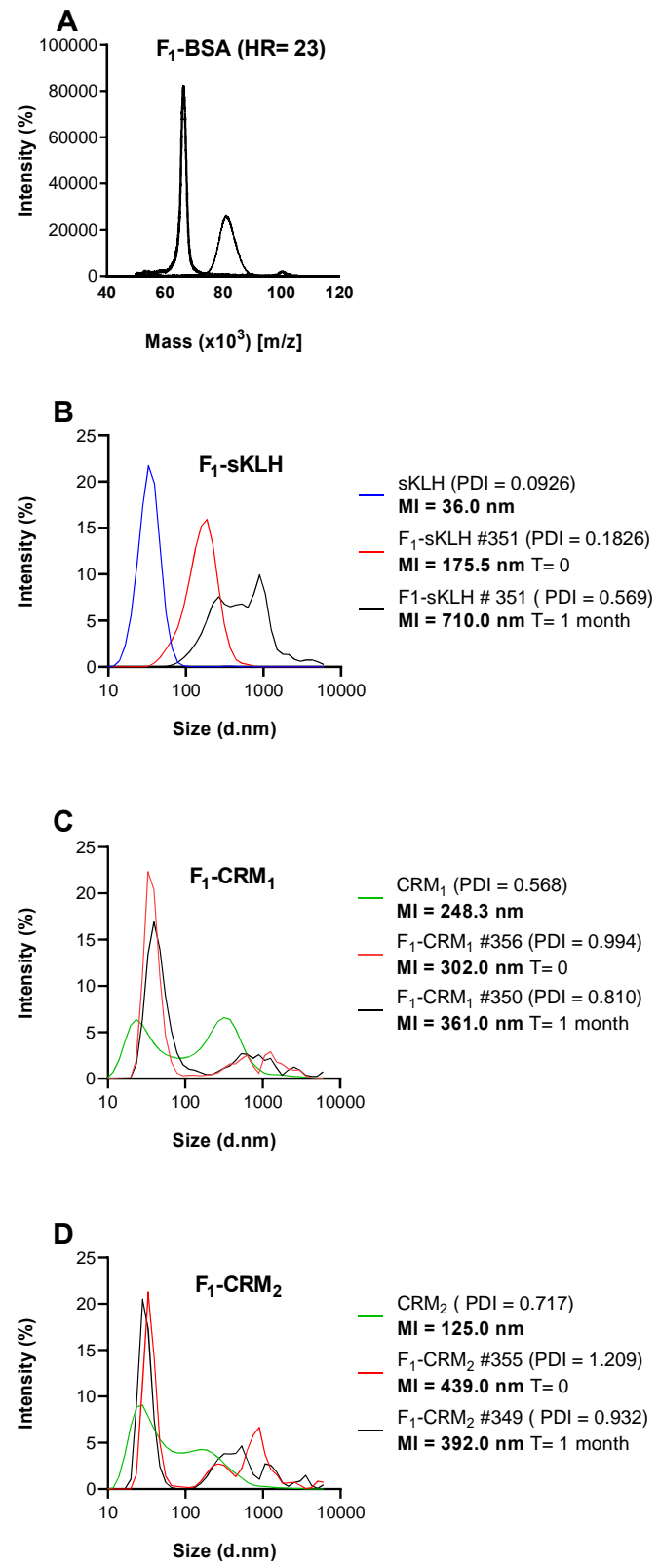
Supplemental Figure 4. Pre-existing immunity to the carrier does not interfere, or minimally interferes with vaccine-induced antibody responses against fentanyl in mice. Male BALB/c mice were first immunized with sKLH, $\mathrm{CRM}_{1}, \mathrm{CRM}_{2}$ or saline as control on day -14 (2 weeks before the first immunization). Then mice were immunized with $\mathrm{F}_{1}-\mathrm{sKLH}, \mathrm{F}_{1}-\mathrm{CRM}_{1}$, and $\mathrm{F}_{1}-\mathrm{CRM}_{2}$ on days 0,14 and 28 , and fentanyl-specific antibodies analyzed at day 35. Previous exposure to sKLH and $\mathrm{CRM}_{2}$ did not affect antibody responses to vaccination. Instead, interference with $\mathrm{F}_{1}-\mathrm{CRM}_{1}$ was detected. Brackets indicated analysis by unpaired t-test to assess effect of pre-immunization with each individual carrier. Symbol: ${ }^{*} \mathrm{p}<0.05$.

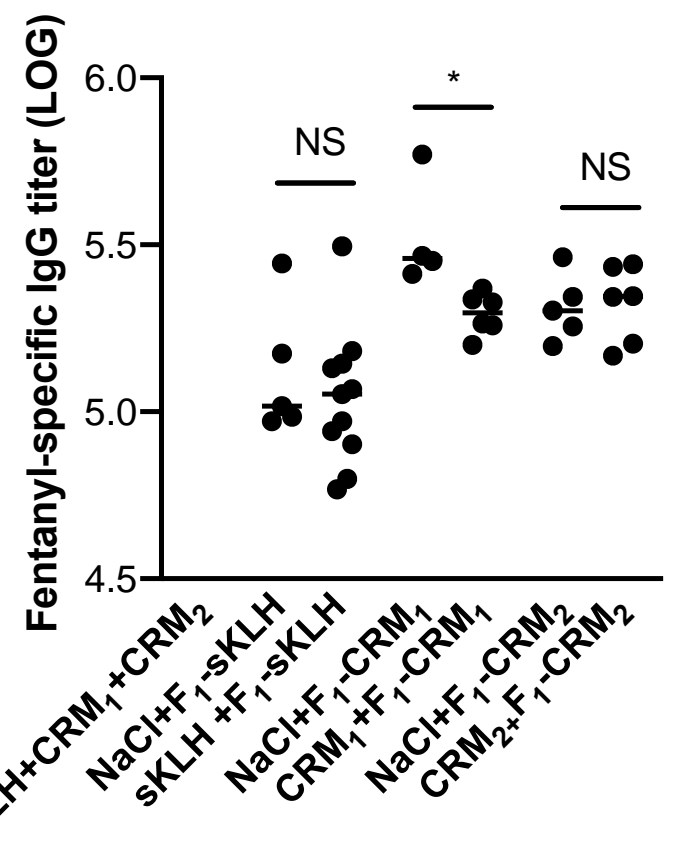


Supplemental Figure 5. Upper panel: structure differences between fentanyl, $F_{1}$ hapten $\left(F_{1}\right)$, and $F_{4}$ hapten $\left(F_{4}\right)$. $F_{1}$ does not contain the $\mathrm{N}$-phenylethyl moiety that is critical for agonist activity at the MOR and displays in its place a tetraglycine peptidic linker. Lower panel: the $F_{1}$ has no functional agonist activity at the MOR. Having haptens that lack the $\mathrm{N}$-phenethyl moiety results in compounds that are devoid of MOR activity. In contrast, the $\mathrm{F}_{4}$ shows activity at MOR.

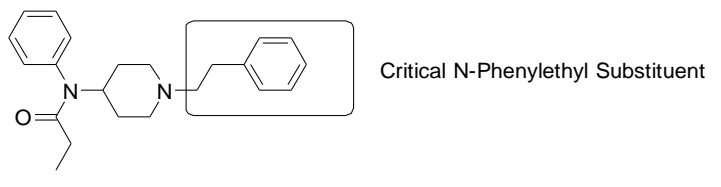

Fentanyl
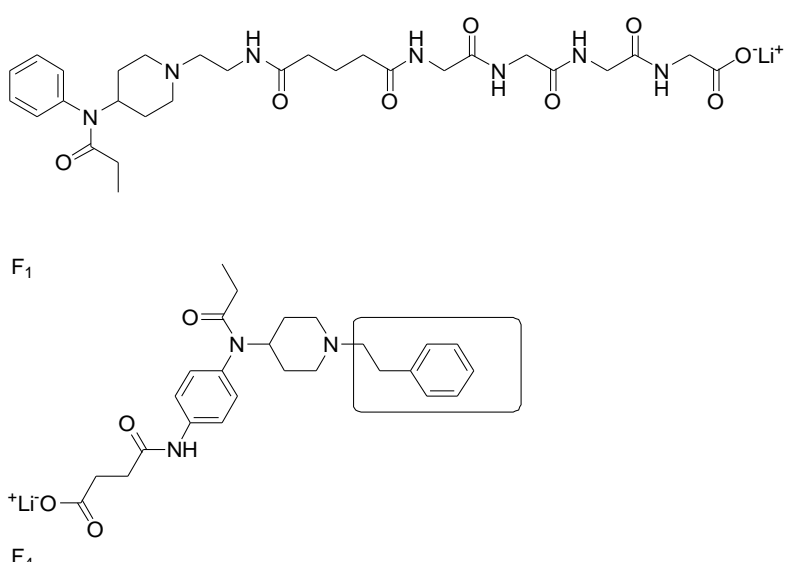

$\mathrm{F}_{4}$
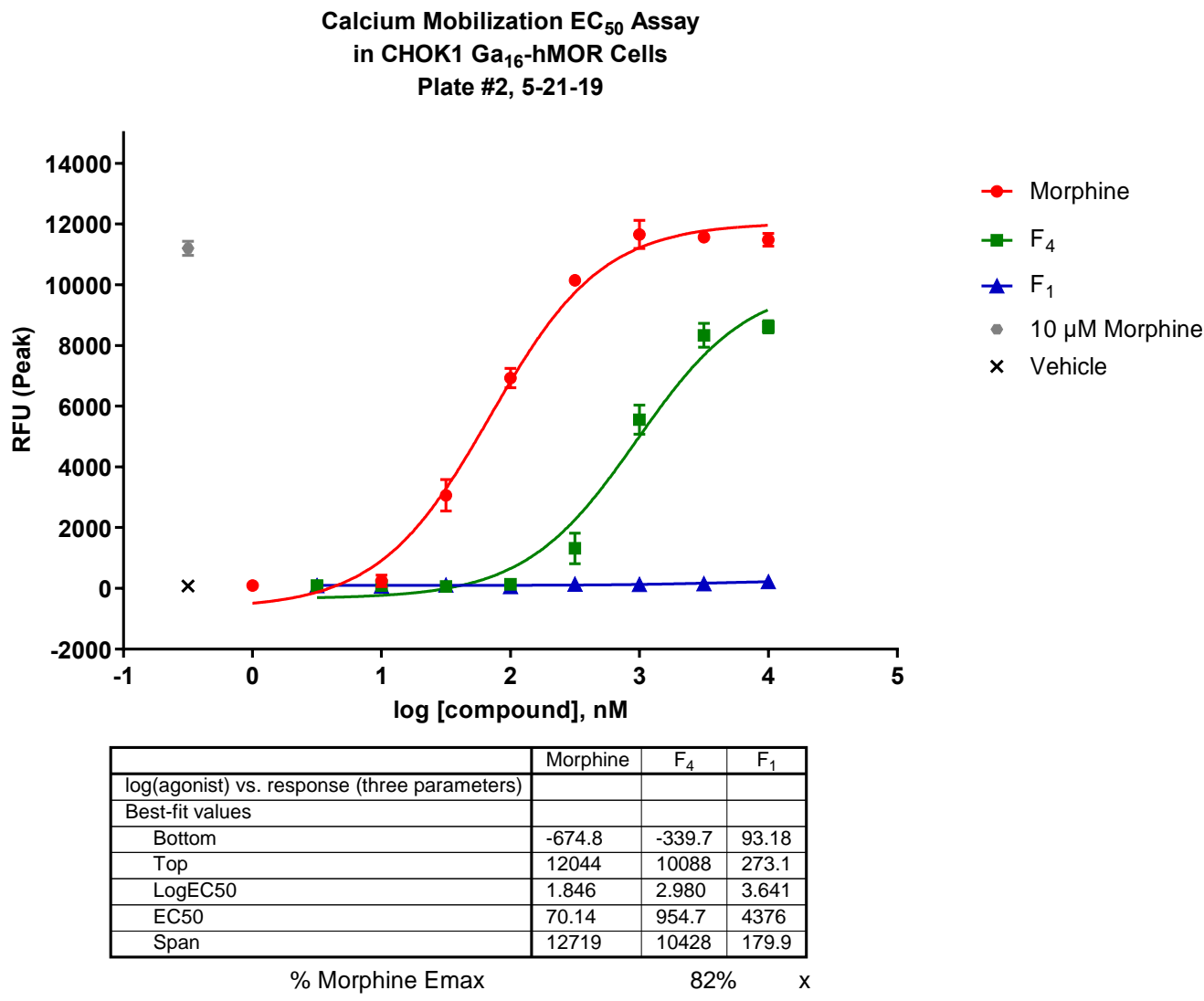
Supplemental Figure 6. Efficacy of vaccines containing haptens $F_{4-6}$ against a high dose of fentanyl in rats. Conjugates containing the $\mathrm{F}_{4-6}$ haptens conjugated to either $\mathrm{SKLH}$ or $\mathrm{CRM}_{2}$ were tested in Sprague Dawley rats $(n=6$, each group). Conjugates were injected i.m. on days $0,21,42$, and 63 . After being challenged with fentanyl and sufentanil $(0.1$ and $0.008 \mathrm{mg} / \mathrm{kg}$, respectively) as shown in Figure 5 , rats were challenged with a final dose of fentanyl ( $1 \mathrm{mg} / \mathrm{kg}$, s.c.) and monitored via pulse oximetry at 15 minutes post-drug challenge. A) Preand post-challenge breath rate, B) breath rate expressed as a percent reduction from baseline, C) brain fentanyl concentrations and D) the ratio of fentanyl in the brain vs. serum as measured by LC/MS. Symbols: ${ }^{* * *},{ }^{* * *},{ }^{* * * *}$ $p \leq 0.05,0.01,0.001,0.0001$ compared to control.
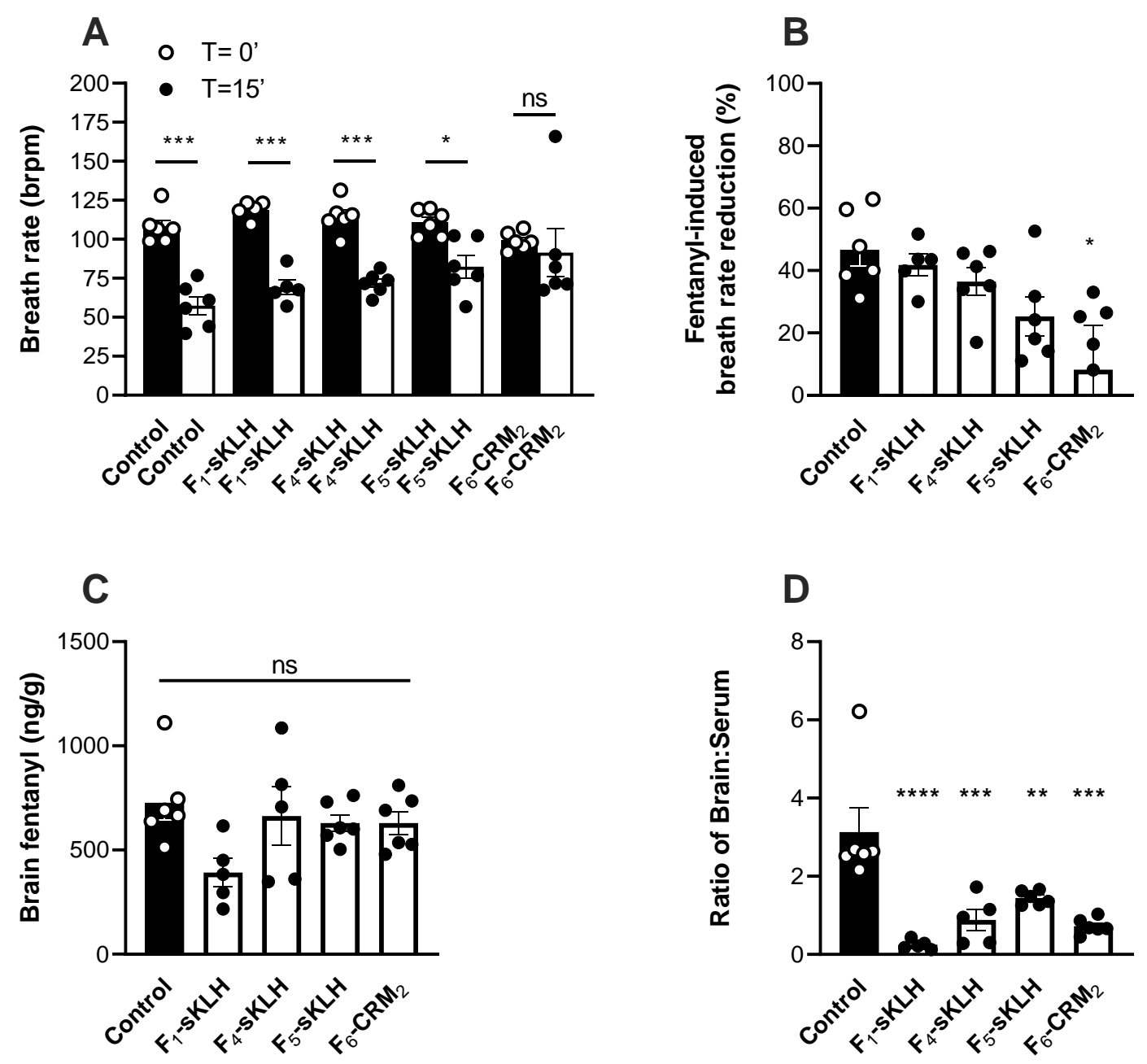

\section{References:}

1. Baehr, C.A., et al., Monoclonal antibodies counteract opioid-induced behavioral and toxic effects in mice and rats. Journal of of Pharmacology and Experimental Therapeutics, 2020. 